\title{
Pathway- and network-based analysis of GWAS data revealed susceptibility gene sets to schizophrenia
}

\author{
Peilin Jia ${ }^{1,2,3}$, Zhongming Zhao ${ }^{1,2,3,4^{*}}$ \\ From UT-ORNL-KBRIN Bioinformatics Summit 2010 \\ Cadiz, KY, USA. 19-21 March 2010
}

\section{Background}

Genome-wide association studies (GWAS) have become a powerful approach to searching for common genetic variants associated with complex diseases. So far, the search for common susceptibility variants has been less successful in psychiatric disorders such as schizophrenia than in many other complex diseases. Among several recent schizophrenia GWA studies, essentially no marker or gene could have achieved genome-wide significance level in any single study [1]. So far, the genetic signal has always been examined at single marker or single gene level in the schizophrenia GWA studies.

\section{Materials and methods}

In this study, we uniquely examined GWAS data at the the gene set level (i.e., pathways and protein-protein interaction (PPI) subnetworks) rather than the SNP level. We first collected a comprehensive list of pathways from the KEGG and BioCarta databases. Additionally, we applied a network module searching approach to search for informative subnetworks in a nodeweighted human PPI network by GWAS markers' $P$ values. Using this module searching method, we identified 100 candidate genes from top ranked schizophrenia-specific modules and used these genes as a gene set for follow up functional enrichment test. We applied two statistical methods (gene set enrichment analysis (GSEA) and hypergeometric test) and also combined them by Fisher's combined method to analyze the gene sets defined by canonical pathways or our module genes. Results were further validated by permutation analysis.

\section{Results and conclusion}

In GSEA analysis, we found the gene set consisting of the network module genes was the most significant among all gene sets, indicating our module searching being efficient in finding the real effect of multiple genes in a more flexible way than classically defined pathways. We also identified a few pathways that are consistently associated with schizophrenia by multiple methods; they included glutamate metabolism pathway, TNFR1 pathway, and TGF beta signaling pathway. These results not only improved our understanding of the underlying pathogenesis of schizophrenia, but also suggested that the gene set based approach is powerful to detect common variants conferring risk to complex diseases.

\section{Author details}

'Department of Biomedical Informatics, Vanderbilt University, Nashville, TN 37232, USA. ${ }^{2}$ Department of Psychiatry, Vanderbilt University, Nashville, TN 37232, USA. ${ }^{3}$ Bioinformatics Resource Center, Vanderbilt University, Nashville, TN 37232, USA. ${ }^{4}$ Vanderbilt-Ingram Cancer Center, Vanderbilt University, Nashville, TN 37232, USA.

Published: 23 July 2010

\section{Reference}

1. Shi J, Levinson DF, Duan J, Sanders AR, Zheng Y, Pe'er I, Dudbridge F, Holmans PA, Whittemore AS, Mowry BJ, et al: Common variants on chromosome 6p22.1 are associated with schizophrenia. Nature 2009, 460(7256):753-757

\section{doi:10.1186/1471-2105-11-S4-O1}

Cite this article as: Jia and Zhao: Pathway- and network-based analysis of GWAS data revealed susceptibility gene sets to schizophrenia. BMC Bioinformatics 2010 11(Suppl 4):O1.

* Correspondence: zhongming.zhao@vanderbilt.edu

'Department of Biomedical Informatics, Vanderbilt University, Nashville, TN 37232, USA 